\title{
المقارنة بين طريقة الإيحائية وطريقة المغناتيسي في تعليم اللغة العربية
}

\author{
Qiya Khaira Hikmatillah \\ UIN Sunan Kalijaga Yogyakarta \\ qiyakhaira@gmail.com
}

\section{Article History:}

Received:

30 November 2020

Revised:

26 Desember 2020

Accepted:

30 Desember 2020

\section{Keywords:}

Method, Hypnoteaching,

Suggestopedia
This study is a comparison of the suggestopedia method and the hypnoteaching method. Sugestopedia is a method that has been initiated for a long time, while bypnoteaching is a method of updating. This research was conducted to determine the advantages and disadvantages of both, so that the updating of the method with the hypnoteaching method can provide learning convenience for students. The method used in this research is descriptive qualitative analysis. The main data sources from this study are the books related to suggestion supported articles and magazines that are in line with the research topic. Therefore this article will explore where the suggestopedia is. In addition to suggestopedia, there is also a bypnoteaching method that uses suggestions and motivation so that students can receive lessons well. Then what is the difference between the two methods. Both of these methods have differences in terms of implementation, but the time required is still the same, which requires a long process to be able to provide suggestions and enjoyment while learning. From a long process and a series of techniques from these two methods it can be seen that these two learning methods are not at all suitable for students who need to learn in a short time, but with a class with a few students, their ability and achievement will be more monitored.

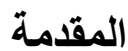

استخدم اللغة العربية أكثر من عشرين بلدان في أنحاء العالم كللغة الأولى في بلادهم، بل استخدم كثير من

مجتمع المسلمين في البلاد اللغة العربية كاللغة الثانية لأنّهم تعلّموا عن دينهم. بالإضـافة إلى ذلك عرفنا أنّ الناس تعلّموا اللغة العربية على غرضين : اللغة العربية كأداة للاتصال أو اللغة العربية كلغة الدين.

وجدت الكاتبة أهم الاهتمام نحو هذه المقالة طريقة الإيحائية هي أسلوب بدأ منذ فترة طويلة، بينما طريقة

التنويم المغناطيسي هي وسيلة للتجديد. تم إجراء هذا البحث لتحديد مز ايا وعيوب كلهمما، بحيث يمكن أن يوفر تحديث الطريقة بطريقة التنويم المفناطيسي راحة التعلم للطلاب. كلتا الطريقتين لهما اختلافات من حيث التنفيذ، لكن الوقت المطلوب لا يزال هو نفسـ، الأمر الذي يتطلب عملية طويلة حتى نتمكن من تقديم الاقتراحات والاستمتاع أثناء التعلم.

ولزاما على اهتمام الطريقة و المدخل و الأسلوب على ما وجدنا الناس تعلّموا اللغة العربية. معنى الطريقة

لغة: السيرة، الحالة، المذهب، الخط في الشيء. و اصتلاحا : مجموعة الأسـاليب التي يتم بوسيطتها تنظيم المجال 
الخارجي للمتعلم، من أجل تحقيق أهداف تربوية معينة.1 مدخل تعلم اللغة العربية هي الطريقة التي تسافر بها حول كيفية تقديم المواد التعليمية والمواد العربية بحيث يتم قبولها واستيعابها و إتقاهها بسهولة من قبل الطلاب بشكل جيد وممتع.2

المدخل هو مجموعة من الافتراضيات المتعلقة بطبيعة تعليم اللغة وتعلمها.3 تقنية التدريس هي طريقة تستخدم لتنفيذ طرق التدريس في الفصل الدراسي. لتجنب الارتباك بسبب تشابه المعنى بين مناهج المصطلحات والنماذج والأساليب والتقنيات، اقترح ريتشارد ورودجرز (1986) نظام تصنيف من ثلاثة أجزاء، أي أنه في الطريقة هناك ثلاثة مستويات، وهي النهج والتصهيم والإجراءات. النهج هو المستوى الأول في الطريقة ، والذي يحتوي على تعريفات للافتراضيات أو المعتقدات أو النظريات حول اللغة وتعلم اللغة، والتي يتم تحديدها عادة في شكل البديهيات (على سبيل المثال: "تتكون اللغة من عدد من الأنماط التي يمكن تعلمها من خلال الحفظ والتكراروالممارسة" أو "تتكون اللغة من العديد من إجراءات الاتصال التي يمكن تعلمها شيئًا فشيينًا من خلال التمارين والممارسات ولعب الأدوار"، وما إلى ذلك). التصميم هو المستوى الثاني في طريقة، والذي يحدد نموذج المنهج (اختيار وترتيب مواد اللغة) و أنواع أنشطة التعلم والتعليم (أنواع التمارين، وكيفية تصنيف الطلاب ، سواء كان استخدام اللغة الشفوية أو المكتوبة ، أو المهام التواصلية). في هذا المستوى الثاني، يتم وصف شكل ووظيفة المواد التعليمية والأنشطة الصفية فيما يتعلق بالمنهج (أو النظرية حول اللغة والتعلم). الإجراء هو المستوى الثالث. الإجراء هو أسلوب تعليهي وممارسـة تستخدم عند تنفيذ الطريقة حيث يكون هذا الإجراء أي شيء مرئي أو مرئي عند تطبيق الطريقة، مثل استخدام بعض سلوكيات التدريس (تسلسل أسئلة وأجوبة واستر اتيجيات تصحيح الخطأ ومقدار الوقت المعطى إلى جو انب معينة من اللغة) ، ونوع المواد السمعية والبصرية ، وأسلوب التفاعل بين المعلم والطلاب.4 الإيحائية (Suggestopaedia) هي نظام تعليمي يستخدم كل الاحتمالات التي يمكن أن يقدمها اقتراح العطاء.5

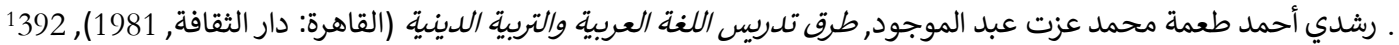

2 Ahmad Izzan, Metodologi Pembelajaran Bahasa Arab (Bandung: Humaniora Utama Press, 2011), 72.

${ }^{3}$ Ahmad Fuad Effendy, Metodologi Pengajaran Bahasa Arab (Malang: Misykat, n.d.), 8.

${ }^{4}$ Syukur Ghazali, Pembelajaran Keterampilan Berbabasa Dengan Pendekatan Komunikatif-Interaktif, 1st ed. (Bandung: Refika Aditama, 2013), 102.

${ }^{5}$ Vienna, DESUGGESTOPAEDLA IS AN ART - CONSTANTLY OSCILLATED ADAPTED ART Summary of Lectures 1995 - 2005 Dr. Georgi Lozanov, 2005, 11.
} 
شهدت الستينات و السبعينات من القرن الميلادي الحالي ظهور العديد من طرق تدريس اللغة التي تؤكد على أهمية مشـاعر الدارس تجاح الوضيع الاجتماعي لغرفة الدراسة. و نظرا لعدم توفر تسمية علمية خاصة لهذه الطرق فسوف أطلق عليها مصطلح "النظرية النفسية الاجتماعية sociopsychological theory". هناك ثلاث طرق تندرج في هذا الاتجاه و هي : الطريقة الصيامتة silent way (جانتو gattegno الاجتماعي community language learning (كوران suggestopedia (لوز الون )، والطريقة الإيحائية l9V^ lozanov ) في التواصل البشري ، عادة ما يكون "في الوسط" هو شكل من أشكال اللغة - يتجسد في الكلام أو الكتابة أو التوقيع أو مزيج من ذلك. ثم هناك ظواهر شبه بارفية مثل تعابير الوجه ، وحركات العين ، والضحك ، والتهد ، والإيماءات ، والمو اقف ، ولغة الجسد الأخرى. في التواصل بوساطة التكنولوجيا ، فإن اللغة واللغة

$$
\text { وهاهي الموضسوعات التدخلان في الوسط الجسـدي للجهازوواجهته6 }
$$

1) Pengaruh Metode Suggestopedia Terhadap Penguasaan Kosakata Bahasa Inggris Siswa Kelas III Sekolah Dasar (Riseu Paulina, Dian Indihadi, Momoh Halimah) ${ }^{7}$

$$
\begin{aligned}
& \text { في المقالة لأولى اهتمت الباحثة بتجربة طريقة الإيحائية لتعلم اللفة الإنجليزية. الطريقة المستخدمة هي } \\
& \text { الطريقة الكمية مع نوع التجربة. استخدمت الباحثة تصميم اختبار قبلي لمجموعة واحدة. توضح نتائج } \\
& \text { البحث أن هناك إتقانًا متز ايدًا لمفردات اللغة .1الإنجليزية في الصف الثالث من مدرسة سيندانج كاسيح } \\
& \text { الثالثة الابتدائية عند مقارنتها قبل وبعد استخدام الاقتراح في تعلم اللغة الإنجليزية. متوسط الدرجات في } \\
& \text { الاختبار التمهيدي هو 47، ٪ بينما متوسط الدرجات في نتيجة ما بعد الاختبار هو 88،5٪. وبالتالي ، هناك } \\
& \text { إتقان متز ايد لمفردات اللغة الإنجليزية في المدرسة الابتدائية في الصف الثالث من سيندانج كاسيح الثالثة } \\
& \text { حول موضوع الأطعمة بعد استخدام الاقتراح بنسبة 41٪. }
\end{aligned}
$$

\footnotetext{
${ }^{6}$ Richard Kern, "Technology as Pharmakon: The Promise and Perils of the Internet for Foreign Language Education," The Modern Language Journal 98, no. 1 (2014): 342, https://doi.org/10.1111/j.1540-4781.2014.12065.x. ${ }^{7}$ Riseu Paulina, Dian Indihadi, and Momoh Halimah, "PENGARUH METODE SUGGESTOPEDIA TERHADAP PENGUASAAN KOSAKATA BAHASA INGGRIS SISWA KELAS III SEKOLAH DASAR," 2015, 9.
} 
2) The Influence of Collaborative Suggestopedia Method And Audio Visual Media Toward Students' Speaking Ability (Dini Deswarni And Setiawati) ${ }^{8}$

و إنما في المقالة الثانية استخدمت هذه الدراسة برنامج المنتجات الإحصائية وحلول الخدمة أي SPSS لتحليل البيانات. تم ربط البيانات باستخدام ارتباط بيرسون لحظية المنتج (SPSS). ثم ، مستوى دلالة 5٪ = 0.05 لأن هذه الدراسة استخدمت اختبار شفهي وتغير كبير F = 0.04 درجة الارتباط بين طريقة الاقتراح والوسائط المرئية والمسسموعة تجاه قدرة الطلاب على التحدث هي 0.689. الفئة بين r 0.5 >0.75 قوية. لذلك ، بناءً على قيمة ارتباط المعامل (r) من الصيغة ، يكون 0.689 قويًا. وأظهرت قبول بديل الفرضية (Ha3). في الختام ، كان لأسلوب الاقتراح التعاوني والوسائط المرئية والمسموعة مساهمة قوية في قدرة الطلاب على

3) The Effect of Suggestopedia Learning Method on Descriptions Writing Skills Of Fourth Grade Elementary Pupils (Rahmasari Dwimarta, St. Y. Slamet, Rukayah) ${ }^{9}$ والمقالة الثالثة يهدف هذا البحث إلى تحديد 1) الفروق في مهارات الكتابة لدى الطالب أثناء استخدام الطريقة الإيحائية والطريقة المباشرة. 2) الفروق في مهارات الكتابة الوصفية لدى الطالب بناءً على دافع التعلم إما مرتفع أو منخفض 3) التفاعل بين طريقة التدريس وطريقة التدريس. يتعلم التلاميذ الدافع. استخدم هذا البحث طرق شبه تجريبية. وخلاصية هذا البحث أن أسلوب التدريس ودافع التعلم لهما تأثير على المهارات الكتابية الوصفية التي حصلت على تفاعل بين دافع التعلم ومهارات التعلم.

4) Suggestopedia Method on Improving Students Reading Comprehension (Vebriana Setia Deny, Syamsul Bahri, Dian Fajrina) ${ }^{10}$

$$
\begin{aligned}
& \text { و الر ابعة، يهدف هذا البحث إلى معرفة ما إذا كانت طريقة الإيحائية قد أدت إلى تحسين فهم الطلاب } \\
& \text { للقراءة. تكونت الاختبارات من } 10 \text { أسئلة متعددة الخيارات للنصيوص السردية. أسلوب تحليل البيانات } \\
& \text { استخدم الباحث المعادلة الإحصائية. وبلغ متوسط درجات الاختبار القبلي (53.18) والاختبار البعدي }
\end{aligned}
$$

\footnotetext{
${ }^{8}$ Dini Deswarni and Setiawati Setiawati, "THE INFLUENCE OF COLLABORATIVE SUGGESTOPEDIA METHOD AND AUDIO VISUAL MEDIA TOWARD STUDENTS' SPEAKING ABILITY," Proceedings of ISELT FBS Universitas Negeri Padang 6, no. 0 (July 4, 2018): 96-107.

${ }_{9}^{9}$ Rahmasari Dwimarta, St Y. Slamet, and Rukayah Rukayah, "The Effect of Suggestopedia Learning Method on Descriptions Writing Skills of Fourth Grade Elementary Pupils," Proceeding of the International Conference on Teacher Training and Education 2, no. 1 (November 23, 2016): 270-76.

10 "Deny, Vebriana Setia, Dkk. Suggestopedia Method on Improving Students Reading Comprehension, Vol.1, No.2, READ, 2016 - Penelusuran Google,” accessed January 6, 2021, h8.
} 
5) Suggestopedia: Pendekatan Pengajaran Bahasa Kedua Yang Bersifat Humanistik (Bambang Eko Hari Cahyono $)^{11}$

والأخر، هذه الدراسة عبارة عن بحث أدبي ، وهو عبارة عن مجموعة توحي بأنه يمكن أن يقال عن طريقة إنسـانية ، لأنها تهتم بالشعور الذي يشعربه الطلاب ، بحيث يمكنها تحسين قدرات التعلم لدى الطلاب.

\section{منهج البحث}

تناقش هذه الدراسة الارتباك حتى الآن ، ويطلق على الإيحائية والتنويم المغناطيسي طريقة أو مدخل أو

أسلوب . و ما الفرق بين كلتا طريقتين. يمكن العثور على هذا في الأدبيات بحيث يكون هذا البحث دراسة أدبية باستخدام طرق التحليل الوصفي. مصادر البيانات الأساسية من هذه الدراسة هي الكتب المتعلقة بالإيحائية و التنويم المفناتيسي المدعومة بالمقالات والمجلات التي تتماشى مع موضيوع البحث. و تأخذ الباحثة من البحث القديم وهو البحث أمى باررة و عبد الكهفي أمر الله ، طريقة التنويم في تعليم اللغة العربية و بعض البحوث عن الطريقة الإيحائية التي ستذكرو تناقش الباحثة في البحث التالي.

\section{نتائج البحث ومناقشتها}

مبدأ الإيحائية وفقًا لثثلاثة مبادئ عامة (سوستيرند غريتون/

$$
\text { ب. ب.يجب أن يتميز التعلم بالفرح وعدم التوتر. }
$$

ج. الإيحائي هووسيلة لاستخدام الاحتياطيات العقلية غير المستخدمة عادة بهدف التعلم¹2.

$$
\text { ثلاث مراحل من الاقتراح : ( ث }
$$

1 ـ يقرأ الطالب الأول المواد من درس اليوم السابق من خلال المحادثة أو اللعبة أو مسرحية هزلية (دراما

قصيرة فكاهية). يتم تجنب تكرار اللغة المتكررة في هذه المرحلة. للتغلب على صعوبات الطلاب ، يتم إعطاء

اسم جديد لكل طالب في الفصل ويطلب منه لعب دور جديد. يُطلب من الطلاب أن يتصرفوا كشخصيات

\footnotetext{
11 "Bambang Eko Hari, Suggestopedia: Pendekatan Pengajaran Bahasa Kedua Yang Bersifat Humanistik, FPBS IKIP PGRI Madiun - Penelusuran Google,” accessed January 6, 2021,

${ }^{12}$ Hadi Kasmaja DS, “THE EFFECTIVENESS OF THE IMPLEMENTATION OF HYPNOTEACHING METHOD TO IMPROVE MOTIVATION AND MATHEMATICS LEARNING RESULT OF CLASS VII STUDENTS AT SMPN 41 BULUKUMBA," Jurnal Daya Matematis 5, no. 1 (March 17, 2017): 8, https://doi.org/10.26858/jds.v5i1.3033.
} 
جديدة ، ونأمل أن يتمكنوا من ممارسة الأصوات التي تكون صعبة من اللغة الهدف بشكل أكثر سلاسـة (لأنه إذا كان خطأ ، فإن الخطأ هو"الشخصية" التي يلعبها ، وليس هو نفسـه. وهنا يكمن "اقتراحه") . 2 ـ ثم يقدم المعلم مادة جديدة من خلال حوارات طويلة مبنية على مو اقف حقيقية. يتم استخدام التقنيات التقليدية مثل تحليل القواعد والترجمة هنا. تم تكوين الحوارات بطريقة تجعل الاستمرارية بين حوارمع آخر من حيث المؤامرة والشخصية والوضع. يستمع الطلاب إلى مدرس موسيقى السامبال يقرأون الحوار ، عادة سطرًا بسطر. تتغير نغمة التجويد التي يغنيها المعلم وتتغير الموسيقى طوال فترة القراءة ، بينما يقرأ الطلاب نص الحوار في كتابهم المجهز بترجمة بلفتهم الأولى. علاوة على ذلك ، يمارس الطلاب أيضًا تقنيات التحكم في التنفس لزيادة تركيزهم عند الاستماع إلى هذا الحوار. ويشار إلى ذلك باسم "حفلة موسيقية نشطة". ثم يستمع الطلاب إلى قراءة الحوار نفسها للمرة الثانية ، وهذه المرة مع عيونهم مغلقة ومر افقة بموسيقى الباروك. وهذا ما يسمى "الحفل السلبي". تم تصميم هاتين المرحلتين ، السلبي والنشط ، لتمكين التعلم الذي يحدث على مستوى اللاوعي ، من خلال استرخاء الطلاب وتقديم مواد ذات صلة وجذابة عاطفية للطلاب.

3 ـ الجلسة الثالثة تسهى مرحلة التفعيل. في هذه المرحلة تعزيزمادة جديدة تمت دراستها في المرحلة الثانية في وقت سـابق. يقوم الطلاب بأداء الأدوار وممارسة اللغة. إذا لزم الأمر ، يقدم المعلم شرحًا للقواعد

$$
\text { باستخدام اللغة الأولى.13 }
$$

التنويم المغناطيسي هو في الواقع القدرة على جلب شخص ما إلى مرحلة التنويم المغناطيسي، حالة المغناطيسي هي حالة سهل قبول الوعي وقبول الاقتراحات أو الاقتراحات المختلفة.14

مفتاح طريقة التنويم المغناطيسي هو كيف يمكن للمدرسين خلق بيئة تعليمية مريحة داخليًا (نفسانيًا) وخارجيًا (جسديًا). لأنه عندما تكون الراحة في التعلم ، سيختبرون أيضًا عملية تعلم ممتعة ، وعندما يكونون في عملية تعليمية مريحة ، يتم التأكد من أن الطلاب سيستوعبون المواد التي يقدمها المعلم بسهولة. يمكن أن يحدث

${ }_{13}$ Ghazali, Pembelajaran Keterampilan Berbahasa Dengan Pendekatan Komunikatif-Interaktif, 100.

14 Ali Akbar Navis, Hypnoteaching (Yogyakarta: Ar-Ruzz Media, 2012), 128-29. 
هذا لأن الظروف المريحة هي ظروف تم إنشاؤها بواسطة عامل التنويم (المعلم) مع اتصال مفيد لجلب موضوع التنويم (الطلاب) إلى حالة اللاوعي.15

$$
\text { خطوات التنويم المغناطيسي في تعلم الرياضيات هي كما يلي: }
$$

(1) التركيز أو التركيز: يمكن تقسيم التنويم المغناطيسي إلى التنويم المغناطيسي الخفيف والتنويم

المغناطيسي العميق. تستخدم عملية التدريس و أنشطة التعلم تقنيات التنويم المغناطيسي الخفيف

بدلاً من التنويم المغناطيسي العميق. إن حالة التركيز عند الدراسة هي في الو اقع حالة يحتاجها الجميع

$$
\text { بحيث لا يتباعد العقل ويصبح المستوى العاطفي للفرد مستقرًا. }
$$

(2) حالة الاسترخاء الجسـدية غالبًا ما يُنسى الاسترخاء أثناء جلسـات التعلم. على الرغم من أن هذا شرط

$$
\text { أسساسي لكي يتمكن الشخص من استيعاب أي بيانات ومعلومات ومعرفة. }
$$

(3) الدعاء و التبصريّ هي قوة لا حصر لها تغلفنا من كل شيء سلبي سيأتي إلينا. تنشط الدعاء القوة

$$
\text { المدفونة في الداخل. }
$$

(4) التأكيد (اقتراح إيجابي) التاكيد هو عبارة إيجابية نضعها في الكتابة والعقل الباطن ، يستخدمها شخص ما لتحديد الأهداف. يجب أن يكون هذا التأكيد إيجابيًا ولا يتعارض مع المعتقدات أو المعتقدات القائمة. يمكن أن تكون التأكيدات على شكل صلوات متكررة تغرس في العقل الباطن ، بحيث يستجيب

$$
\text { هل الإيحائية والتنويم المنفناطيسي طريقة أو المدخل أو الأسلوب؟ }
$$

كثير من المدرسين يتحيرون في تفريق بين الطريقة و المدخل ووالأسلوب ـ. وتأكيد "الإيحائية " أ هي الطريقة أو المدخل أو الأسلوب. قد عرضنا البيان في المقدمة الفرق بين الطريقة و المدخل و الأسلوب. و لهذا، الإيحائية والتنويم المغناطيسي هي الطريقة، فمن الطريقة توجد ثلاث مستويات، المستوى الأول هو المدخل: في عملية التعلم ، يستخدم الطلاب عقلًا فاقدًا للوعي من خلال تقديم اقتراح إيجابي لجعل الطلاب يسترخيون ويتجمعون. يتم استخدام إيقاع الموسيقى في عملية التعلم التعليمية. يطبق هذا الأسلوب تأثير اقتراح

\footnotetext{
${ }^{15}$ Kasmaja DS, “THE EFFECTIVENESS OF THE IMPLEMENTATION OF HYPNOTEACHING METHOD TO IMPROVE MOTIVATION AND MATHEMATICS LEARNING RESULT OF CLASS VII STUDENTS AT SMPN 41 BULUKUMBA," 108.

16 Kasmaja DS, 108-9.
} 
السلوك البشري.17 المستوى الثاني هو التصيميم والذي يحدد نموذج المنهج (اختيار وترتيب مواد اللغة) و أنواع أنشطة التعلم والتعليم (أنواع التمارين ، وكيفية تصنيف الطلاب ، سواء كان استخدام اللغة الشفوية أو المكتوبة ، أو المهام التواصلية). يركز طلب نجاح طريقة الإيحائية على عدد الكلمات التي يمكن إتقانها بحيث تحصل المفردات على التركيز الرئيسي ، ويتم وضيع القواعد بشكل صريح ولكن في الحد الأدنى. سيتعلم المتعلمون بشكل أفضل إذا لم يوجه انتباههم الواعي إلى أشكال اللغة ، ولكن إلى استخدام اللغة ، ويمكنهم التحدث بشكل تواصل. يقرأ المتعلمون أيضًا اللغة الهدف (على سبيل المثال الحوار) ويكتبون (على سبيل المثال كتابة المقالات). يتم إجراء دروس الاقتراح في لوز انوف في معهد علم الإقتراحات في صوفيا ، بلغاريا. يتكون الفصل من مجموعة صغيرة تتكون من حوالي ب طالبًا لمدة ع سـاعات بشكل مكثف كل يوم من الشهر. تتكون كل سـاعة من ثلاثة أجزاء: أ. يتم التكرار (المراجعة) من خلال المحادثة (الوثيقة السادة) أو الألعاب أو لعب الأدوار. لا يتم استخدام مختبرات اللغة في هذا القسم. يمكن إجراء التدريب والتصحيح فقط.

ب. يعتمد تقديم المواد الجديدة على المو اقف المألوفة. تتضهمن هذه المواد حوارًا مطولًا من حوالي . إلى عا صفحة عن طريق إضافة تفسيرات وترجمات نحوية مهمة.18

باستخدام الطريقة الإيحائية ، يمكن للمعلمين تحفيز طلاهم على التحدث ومساعدتهم على محاربة مخاوفهم وقلقهم في التحدث عن طريق إزالة كتلهم العقلية.19 كما تقول إيئيك حكمة الهداية في بحثها، أن سورة لقمان علمت الكثير عن مفهوم التنويم المفناطيسي. ويمكن ملاحظة ذلك من طريقة تواصل لقمان وتعليم أطفاله شيئًا ما. إحدى الطرق هي استدعاء طفله بحنان تام واستخدام مكالمات النداء اللطيفة. و مع ذلك يمكن أن يفتح هذا المجال الحرج من طفله ، بحيث عندما يتم فتح المجل الحرج سيتم قبول الإيحائي المقدم بسهولة.20 للاهتمام مع إضـاءة كافية وهواء21

\footnotetext{
17 "Deny, Vebriana Setia, Dkk. Suggestopedia Method on Improving Students Reading Comprehension, Vol.1, No.2, READ, 2016 - Penelusuran Google," 131. 18 "METODE PEMBELAJARAN BAHASA ARAB KOMUNIKATIF (METODE SUGGESTOPEDIA DAN METODE COMMUNITY LANGUAGE LEARNING) - PDF Download Gratis," 204-5, accessed January 6, 2021, https://docplayer.info/32350139-Metode-pembelajaran-bahasa-arab-komunikatif-metode-suggestopedia-danmetode-community-language-learning.html.

${ }^{19}$ Kusnia, Windi. NIM. 2813133121. 2017. The Effectiveness of Sugestopedia Method to Improve Students' Speaking Ability At Mts Al-Huda Bandung In Academic Year 2016/2017. Skripsi. Tadris Bahasa Inggris , Fakultas Tarbiyah dan Ilmu Keguruan, Institute Agama Islam Negeri (IAIN) Tulungagung. h. I ${ }^{20}$ Iik Hikmatul Hidayat, "JURUSAN PENDIDIKAN AGAMA ISLAM FAKULTAS ILMU TARBIYAH DAN KEGURUAN UINIVERSITAS ISLAM NEGERI SYARIF HIDAYATULLAH JAKARTA 2019,” n.d., 62.

${ }^{21}$ Muhammad Amin Rasyid, Teaching English as a Foreign Language in Indonesia: Theory, Practice, and Research (Ujung Pandang: FBS IKIP Ujung Pandang, 1997), 206.
} 


$$
\text { يتم تقديم الموضوع في مرحلتين رئيسيتين ، بمعنى }
$$

(1) مرحلة التقبل، حيث يقرأ المعلم الموضيوع على شكل حوار ، يضبط صهوته حسب الإيقاع ثم يتبعه الطالب أو

$$
\text { يقلده أو يستمع إليه ثم يقرأه في المنزل ؛ }
$$

(r) مرحلة التنشيط، حيث يتم دعوة الطلاب أو المشاركة في أنشطة مختلفة مصممة لمساعدتهم على الاستفادة من التسهيلات بمواد جديدة. وتشمل هذه الأنشطة الدراما والألعاب والغناء وتمارين الأسئلة والأجوبة.

يتم التدريس في غرفة ممتعة للغاية ومرضية للغاية. هذا يعني خلق بيئة تعليمية مثلى. تعرض الجمل الإيجابية المنشورة على الحائط أو الملصقات المنشورة معلومات نحوية للغة الهدف بقصيد إفادة الطلاب. 22 أ. تشمل نقاط القوة في هذه الطريقة ما يلي:

1) يمكن أن تعزز هذه الطريقة استمتاع الطلاب بالشخصية الخيالية التي يلعبونها، مع أسلوب المعلم غير

التقييهي، ومع المواد التعليمية المثيرة للاهتمام ، بما في ذلك استخدام الموسيقى الكلاسيكية.

2) يمكن أن تؤدي استمرارية الحوارات المستخدمة وطولها إلى تزويد الطلاب بفاعلية بالعالم الخيالي الذي

$$
\text { يمكنهم الارتجال فيه. }
$$

3) العدد الصغير للمشـاركين في الفصل (يفضل أن يكون بحد أقصى 12) يولد جوًا مريحًا كما لو لم يكن في

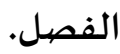

4) يمكن للطلاب تعزيز مشاعر التعاون القوية بينهم لأنهم يساعدون بعضهم البعض على استيعاب جميع

$$
\text { ب. تشمل عيوب هذه الطريقة: الدروس التي تلقوها. }
$$

1) يمكن استخدامه فقط للمجموعات الصغيرة (بحد أقصى 12 شخصًا).

2) يجب أن تكون مجهزة بعدد من المر افق والبنية التحتية المناسبة ، وبالطبع سيكون من المكلف إدارتها. 3) على الرغم من أهها فريدة من نوعها ، فإن عرض المواد التي تعتمد في الغالب على القواعد الهيكلية يعطي

الانطباع بأن هذه الطريقة لا تختلف كثيرًا عن الطرق السابقة الأخرى 23

22 Ghazali, Pembelajaran Keterampilan Berbahasa Dengan Pendekatan Komunikatif-Interaktif, 153.

${ }^{23}$ Ghazali, 160-61.

Program Studi Pendidikan Bahasa Arab Universitas Yudharta Pasuruan https://jurnal.yudharta.ac.id/v2/index.php/studi-arab 
بصرف النظرعن نقاط الضعف أو الضعف في هذه الطريقة ، ولكن هناك العديد من الأسباب التي يمكن استخدامها كأسـاس لتطبيق هذه الطريقة المقترحة في تعلم اللغة ، وخاصة اللغات الأجنبية ، بما في ذلك: 1. يمكن أن تؤدي خدمة الإيحائية إلى عملية تعليم وتعلم ممتعة لأن أحد مبادئها هو متعة وتعتبر شيئًا سهلاً. هذا مطلوب في تعلم اللغة (بما في ذلك اللغة العربية) ، يجب أن يكون تعلم اللغة في جو ممتع وممتع. 2. تعتبر هذه الطريقة الفرد كإنسان كامل حيث تتكامل القوة الجسدية والذوق والروح والفكر في عملية التعلم. التركيزهو قوة المنطق بالإضافة إلى إحساس الفرد بالذوق.

3. أحد العناصر المهمة في هذه الطريقة هووجود تفويض من المعلم يوضح أن المعلم يجب أن يكون مؤهلاً وأن يكون

$$
\text { مؤهلاً ، سواء في اللغويات أو الطب النفسي أو المادي.24 }
$$

هناك العديد من الفوائد التي يمكن تحقيقها من خلال تطبيق التنويم المغناطيسي في التعلم داخل

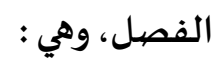

(1) يصبح التعلم ممتعًا و أكثر متعة الطلاب ، وكذلك للمعلمين.

(2) يمكن أن يجذب التعلم انتباه الطلاب من خلال إبداعات اللعبة المختلفة التي يطبقها المعلم.

$$
\text { (3) أصبح المدرسون أكثرقادرة على إدارة عواطفهم. }
$$

(4) التعلم يمكن أن ينمو علاقة منسجمة بين المعلمين والطلاب.

(5) يستطيع المعلم التغلب على الطلاب الذين يجدون صعوبة في التعلم من خلال نهج شخصي.

(6) يمكن للمعلمين تعزيزحماس الطلاب للتعلم من خلال ألعاب التنويم المغناطيسي.

(7) يساعد المعلم الطلاب في التخلص من العادات السيئة مما يملكون قبل25

$$
\text { بين التنويم المغناطيسي و الإيحائية }
$$

التنويم المغناطيسي هو أحد طرق التعليم الجديدة التي تم تطويرها من استخدام العلاج بالتنويم المغناطيسي لأغراض الصحة وعلم النفس وزيادة الدافع للجنود في الماضي. تدريجياً ، اخترق العلاج بالتنويم المغناطيسي عالم التعليم ، الذي يسنى بالتنويم المغناطيسي ، كتحسين للطريقة الكلاسيكية السابقة. يمكن تعلم اللغة العربية باستخدام أساليب التنويم المغناطيسي عن طريق الشروع دائمًا في التعلم من خلال توفير الحافز ،

\footnotetext{
24 "METODE PEMBELAJARAN BAHASA ARAB KOMUNIKATIF (METODE SUGGESTOPEDIA DAN METODE COMMUNITY LANGUAGE LEARNING) - PDF Download Gratis," 207.

${ }^{25}$ Yuatisia N, Hypnoteaching: Seni Mengeksplorasi Otak Peserta Didik (Yogyakarta: Ar-Ruzz Media, 2012), 79.
} 
والمعادلة بين المو اقف ، والحركات ، واللغة ، وموجات الدماغ بين الطلاب ، ثم المضبي في توجيه الطلاب لإجراء التعلم

وفقًا لتعليمات المعلم.26

طبقات مجموعات التفكير المرتبطة بموجات الدماغ والتي يمكن اكتشافها هي: بيتا ، ألفا ، ثيتا ، دلتا. يمكن

قياس موجات بيتا ، وهي 13-40 موجة في الثانية. بينما ألفا هي 8-13 موجات في الثانية. فكلما قل عدد الموجات ، زاد استرخاء دماغنا وزادت حشودنا الذهنية. ثيتا ، مع المعدات الحديثة يمكننا قياس هذه الموجات بقدر4-7 موجات في الثانية. دلتا: 2/1 - 4 دورات في الثانية. 27

من المعروف أنّ الإيحائي و التنويم المغناطيسي يقدم على حدٍ سواء اقتراحات جيدة للطلاب من حيث التعلم ، مما يميز أن الإيحائي الاقتراح باستخدام الموسيقى كأداة لجعل الجو أكثر استرخاء.

قال ضافي في مقالة ويندا أنّ أنواع الموسيقى الهادئة (الموسيقى الهادئة) يمكن أن تجعل الجو الهدوء

والانسجام في البيئة ، بحيث يمكن أن تجلب آثار إيجابية لتطوير إدراك المستمع ، بصرف النظر عن أن بعض الموسيقى يمكن تنتج أمزجة للإنتاجية البشرية تسهى المنبهات الخاصة لحاسة السمع.28

دجوهان يجادل في مقالة ويندا بأن هناك ثلاثة مفاهيم رئيسية فيما يتعلق بالتأثير الموسيقى ، وهي:

الموسيقى مهمة لأنها شيء جيد ، فهذا يعني أن الموسيقى مع بعض الخصيائص الإيجابية التي يمكن أن

يكون لها تأثير إيجابي على المستمع. لذا يمكن أن يكون للموسيقى تأثير إيجابي و سلبي لمستمع الموسيقى.

الموسيقى جزء من الحياة كما أنها جزء من الجمال الثقافي البشر ، بالإضافة إلى وجود قيم إيجابية

مفيدة للغاية. الدماغ البشري والتي منذ الولادة مجهزة بالقدرة على الفهم والمعالجة الموسيقى

والصوت دليل على أن الموسيقى جزء منها الحياة.

من خلال تطوير القدرات الموسيقية ، سيكون التميز الفو ائد التي تر افقه. ممارسة والاستماع

والأنشطة سيعزز تقييم الموسيقى التطور المعرفي والجسـدي والعاطفي ،والاجتماعية.29

\footnotetext{
${ }^{26}$ R. Umi Baroroh and Abdul Kahfi Amrulloh, "Hypnoteaching Method in Arabic Learning," Jurnal Al Bayan: Jurnal Jurusan Pendidikan Bahasa Arab 11, no. 1 (June 1, 2019): 145, https://doi.org/10.24042/albayan.v11i1.3634.

27 anand krishna, NeoSpiritual Hypnotherapy (Jakarta: Gramedia Pustaka Utama, 2012), 136.

${ }^{28}$ Murliansyah, Wendi Alhimda, Efektivitas Metode Suggestopedia Menggunakan Musik Klasik Terhadap Minat

Belajar Bahasa Inggris Pada Siswa Smpn 13 Yogyakarta, UNNES, Semarang, 2013 : h.40

${ }^{29}$ murliyansyah, h.41
}

Program Studi Pendidikan Bahasa Arab Universitas Yudharta Pasuruan https://jurnal.yudharta.ac.id/v2/index.php/studi-arab 
الإيحائية هي طريقة تتضمن المدخل والتصيميم وأسلوب التعلم. وبنقاط التشابه أيضًا بين التنويم

المغناطيسي الإيحائية ، الإيحائية هي التنويم المفناطيسي باستخدام الموسيقى الكلاسيكية بحيث يمكن للاقتراحات

أن تدخل روح الطلاب بطريقة مريحة للغاية. هذا الإيحائي مليء بالدفاع والدروس بالموسيقى ، لذلك يمكننا أن نعرف أن الطريقة الإيحائية تصبح وحدة تعليمية موحدة مع الموسيقى ، كما هي موضحة في المناقشة أن العديد يستخدمون الموسيقى كأداة تعليمية.

الموسيقى المستخدمة هي موسيقى الباروك ، وهي ناعمة ويمكن أن تكون مهدئة ، تمامًا كما هو الحال عندما

يكون الطالب من العصاميين أو التعلم الفردي وهو يبحث عن موقف هادئ أو حتى أثناء الاستماع إلى أغنية أو موسيقى مرغوبة .

نرى من الخطوة بين هذه الطريقتين أن هناك اختلافًا لأن اقتراحو بيديا يضع اقتراحات في بداية التعلم بالموسيقى بينما يتضيمن التنويم المغناطيسي اقتراحات في نهاية التعلم. فيما يتعلق بفو ائد هاتين الطريقتين ، تم اختيار الطلاب ليشعروا بالراحة والاستمتاع بالدرس. لا يزال من الممكن استخدام التنويم المغناطيسي فقط مع الفصل الذي يضهم عددًا كبيرًا من الطلاب ، بينما الاقتراح عبارة عن فصبل يضِم عددًا أقل من الطلاب. ومع ذلك ، لا يمكن إجراء هاتين الطريقتين مرة واحدة فقط ، فهي ضرورية بشكل متكرر للاقتراحات التي تصبح عقلية للطلاب.

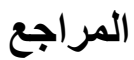

"Bambang Eko Hari, Suggestopedia: Pendekatan Pengajaran Bahasa Kedua Yang Bersifat Humanistik, FPBS IKIP PGRI Madiun - Penelusuran Google.” Accessed January 6, 2021.

Baroroh, R. Umi, and Abdul Kahfi Amrulloh. "Hypnoteaching Method in Arabic Learning." Jurnal Al Bayan: Jurnal Jurusan Pendidikan Bahasa Arab 11, no. 1 (June 1, 2019): 133-48. https://doi.org/10.24042/albayan.v11i1.3634.

"Deny, Vebriana Setia, Dkk. Suggestopedia Method on Improving Students Reading Comprehension, Vol.1, No.2, READ, 2016 - Penelusuran Google.” Accessed January 6, 2021.

Deswarni, Dini, and Setiawati Setiawati. "THE INFLUENCE OF COLLABORATIVE SUGGESTOPEDIA METHOD AND AUDIO VISUAL MEDIA TOWARD STUDENTS' SPEAKING ABILITY.” Proceedings of ISELT FBS Universitas Negeri Padang 6, no. 0 (July 4, 2018): 96-107.

Dwimarta, Rahmasari, St Y. Slamet, and Rukayah Rukayah. "The Effect of Suggestopedia Learning Method on Descriptions Writing Skills of Fourth Grade Elementary Pupils." Proceeding of the International Conference on Teacher Training and Education 2, no. 1 (November 23, 2016): 270-76.

Effendy, Ahmad Fuad. Metodologi Pengajaran Bahasa Arab. Malang: Misykat, n.d.

Ghazali, Syukur. Pembelajaran Keterampilan Berbahasa Dengan Pendekatan Komunikatif-Interaktif. 1st ed. Bandung: Refika Aditama, 2013. 
Hidayat, Iik Hikmatul. "JURUSAN PENDIDIKAN AGAMA ISLAM FAKULTAS ILMU TARBIYAH DAN KEGURUAN UINIVERSITAS ISLAM NEGERI SYARIF HIDAYATULLAH JAKARTA 2019," n.d., 62.

Izzan, Ahmad. Metodologi Pembelajaran Bahasa Arab. Bandung: Humaniora Utama Press, 2011.

Kasmaja DS, Hadi. "THE EFFECTIVENESS OF THE IMPLEMENTATION OF HYPNOTEACHING METHOD TO IMPROVE MOTIVATION AND MATHEMATICS LEARNING RESULT OF CLASS VII STUDENTS AT SMPN 41 BULUKUMBA.” Jurnal Daya Matematis 5, no. 1 (March 17, 2017): 103. https://doi.org/10.26858/jds.v5i1.3033.

Kern, Richard. "Technology as Pharmakon: The Promise and Perils of the Internet for Foreign Language Education.” The Modern Language Journal 98, no. 1 (2014): 340-57. https:// doi.org/10.1111/j.1540-4781.2014.12065.x.

krishna, anand. NeoSpiritual Hypnotherapy. Jakarta: Gramedia Pustaka Utama, 2012.

"METODE PEMBELAJARAN BAHASA ARAB KOMUNIKATIF (METODE SUGGESTOPEDIA DAN METODE COMMUNITY LANGUAGE LEARNING) - PDF Download Gratis." Accessed January 6, 2021. https://docplayer.info/32350139-Metode-pembelajaran-bahasa-arabkomunikatif-metode-suggestopedia-dan-metode-community-language-learning.html.

N, Yuatisia. Hypnoteaching: Seni Mengeksplorasi Otak Peserta Didik. Yogyakarta: Ar-Ruzz Media, 2012. navis, ali akbar. Hypnoteaching. Yogyakarta: Ar-Ruzz Media, 2012.

Paulina, Riseu, Dian Indihadi, and Momoh Halimah. "PENGARUH METODE SUGGESTOPEDIA TERHADAP PENGUASAAN KOSAKATA BAHASA INGGRIS SISWA KELAS III SEKOLAH DASAR," 2015, 9.

Rasyid, Muhammad Amin. Teaching English as a Foreign Language in Indonesia: Theory, Practice, and Research. Ujung Pandang: FBS IKIP Ujung Pandang, 1997.

Vienna. DESUGGESTOPAEDIA IS AN ART - CONSTANTLY OSCILLATED ADAPTED ART Summary of Lectures 1995 - 2005 Dr. Georgi Lozanov, 2005.

محمد عزت عبد الموجود, رشدي أحمد طعمة. طرق تلربس اللغة العربية والتتربية الدينية. القاهرة: دار الثقافة, 1981 
114 | Volume 11, Nomor 2, Desember 2020

Program Studi Pendidikan Bahasa Arab Universitas Yudharta Pasuruan https://jurnal.yudharta.ac.id/v2/index.php/studi-arab 